\title{
Hydrogen-assisted fatigue crack propagation in a pure BCC iron. Part I: Intergranular crack propagation at relatively low stress intensities
}

\author{
Yuhei Ogawa ${ }^{1,2^{*}}$, Domas Birenis $^{3}$, Hisao Matsunaga ${ }^{4,5,6}$, Osamu Takakuwa ${ }^{4,5}$, Junichiro Yamabe ${ }^{7,8}$, \\ Øystein Prytz ${ }^{3}$ and Annett Thøgersen ${ }^{9}$ \\ ${ }^{1}$ Graduate School of Engineering, Kyushu University, 744 Motooka, Nishi-ku, Fukuoka 819-0395, Japan \\ ${ }^{2}$ Research Fellow of the Japan Society for the Promotion of Science, 744 Motooka, Nishi-ku, Fukuoka 819-0395, Japan \\ ${ }^{3}$ Department of Physics, Centre for Materials Science and Nanotechnology, University of Oslo, PO Box 1048 - Blindern, NO-0316 \\ Oslo, Norway \\ ${ }^{4}$ Department of Mechanical Engineering, Kyushu University, 744 Motooka, Nishi-ku, Fukuoka 819-0395, Japan \\ ${ }^{5}$ Research Center for Hydrogen Industrial Use and Storage (HYDROGENIUS), Kyushu University, 744 Motooka, Nishi-ku, Fukuoka \\ 819-0395, Japan \\ ${ }^{6}$ International Institute for Carbon-Neutral Energy Research (I2CNER), Kyushu University,744 Motooka, Nishi-ku, Fukuoka 819- \\ 0395, Japan \\ ${ }^{7}$ AIST-Kyushu University Hydrogen Materials Laboratory (HydroMate),744 Motooka, Nishi-ku, Fukuoka 819-0395, Japan \\ ${ }^{8}$ Department of Mechanical Engineering, Fukuoka University, 8-19-1 Nanakuma, Jonan-ku, Fukuoka 814-0180, Japan \\ ${ }^{9}$ SINTEF Materials and Chemistry, PO Box 124 - Blindern, NO-0314 Oslo, Norway
}

\begin{abstract}
The role of hydrogen on intergranular (IG) fracture in hydrogen-assisted fatigue crack growth (HAFCG) of a pure iron at low stress intensity was discussed in terms of the microscopic deformation structures near crack propagation paths. The main cause of IG fracture was assumed to be the hydrogen-enhanced dislocation structure evolution and subsequent microvoids formation along the grain boundaries. Additionally, the impact of such IG cracking on the macroscopic FCG rate was evaluated according to the dependency of IG fracture propensity on the hydrogen gas pressure. It was first demonstrated that the increased hydrogen pressure results in the larger area fraction of IG and corresponding faster FCG rate. Moreover, gaseous hydrogen environment also had a positive influence on the FCG rate due to the absence of oxygen and water vapor. The macroscopic crack propagation rate was controlled by the competition process of said positive and negative effects.
\end{abstract}

\section{Introduction}

For the storage and transportation of compressed gaseous hydrogen in the forthcoming hydrogen energybased society, hydrogen-assisted fatigue crack growth (HAFCG) in structural steels is of significant concern with regards to the safe design of high-pressure components such as pressure vessels or pipelines $[1,2]$. For elucidating the detailed fatigue crack growth (FCG) behaviour of iron and steels with ferritic, i.e. bodycentered cubic (BCC) crystal structure under the presence of hydrogen, we conducted FCG tests of a commercially pure iron in gaseous hydrogen environment with various pressures at room temperature. Our experiments revealed that the FCG behavior in gaseous hydrogen environment was characterized by two distinctive parts, i.e. the regime with no or slight acceleration of FCG compared to in air at relatively low stress intensities, and the highly accelerated regime at higher stress intensities, both of which will be referred to in the following manuscript as Stage I and II regimes, respectively. Additionally, hydrogen significantly altered the fracture surface morphologies in both Stage I and II FCG regimes. Whereas the large FCG acceleration in Stage II regime was accompanied by transgranular (TG) quasi-cleavage (QC) type fracture surfaces, brittle appearance intergranular (IG) features were observed in Stage I, contrary to the fact that there was no significant change of macroscopic FCG rate in this FCG regime. The main focus of this paper is the underlying mechanism dominating such hydrogen-induced IG crack propagation in Stage I FCG regime and its role on the macroscopic FCG acceleration. The details of Stage II HAFCG will be explained in the second part (Part II) of this series of papers presented by Birenis et al. [3].

While several researchers have also reported similar alteration of fracture features in hydrogen gas in the past, i.e. appearance of $\mathrm{IG}$ and $\mathrm{QC}$ with regards to low carbon or low alloy commercial steels $[1,4,5]$, most of the interest had been particularly focused on the Stage II FCG with QC type fracture [5-7]. However, because the large part of fatigue life in practical components is

Corresponding author: yuhei.ogawa.bz@,gmail.com 
generally accounted for by the crack propagation under low stress intensity, the Stage I FCG accompanied by IG fracture should not be depreciated when attempting to evaluate the overall HAFCG phenomena.

In this study, a combination work of various observation techniques including electron backscattered diffraction (EBSD), electron channelling contrast imaging (ECCI) and transmission electron microscopy (TEM) were applied to the crack propagation paths in hydrogen gas. The role of hydrogen in triggering the fracture along grain boundaries (GB) is then discussed based on the amount of plastic deformation and dislocation structures underneath the IG fracture surfaces.

\section{Material and Experiments}

A hot-rolled commercially pure iron (JIS-C2504) plate with the chemical composition of $0.001 \% \mathrm{C}-0.07 \% \mathrm{Mn}-$ $0.010 \% \mathrm{P}-0.003 \% \mathrm{~S}$ was used in this study. The yield stress, $\sigma_{\mathrm{y}}$ and ultimate tensile strength, $\sigma_{\mathrm{B}}$, measured in air were 133 and $252 \mathrm{MPa}$, respectively. For the FCG tests, compact-tension (CT) specimens with a width, $W$, of $50.8 \mathrm{~mm}$ and a thickness, $B$, of $10.0 \mathrm{~mm}$ were cut out from the L-T orientation of the hot-rolled plate.

The FCG tests were conducted in air, 0.7 MPa nitrogen gas (purity of $>99.999 \%$ ) and hydrogen gas with the pressures of $0.2,0.7$ and $20 \mathrm{MPa}$ (purity of $>99.999 \%$ ) at room temperature, according to the ASTM-E647 standard [8]. The constant load range condition $(\triangle P$ constant tests) was used for obtaining the FCG curves in each testing environment. On the other hand, the tests were also conducted under constant stress intensity factor range, $\Delta K\left(\Delta K\right.$-constant tests, $\left.\Delta K=12 \mathrm{MPa} \cdot \mathrm{m}^{1 / 2}\right)$ in air and $0.7 \mathrm{MPa}$ hydrogen for analyzing the crack propagation behavior under a specific $\Delta K$ value. The crack length during the FCG tests were measured by unloading the elastic compliance method with the compliance measured with a clip-on gauge. The load ratio, $R$ of 0.1 was chosen for all the experiments, and the testing frequency was $1 \mathrm{~Hz}$ for tests in air and hydrogen, and $5 \mathrm{~Hz}$ for the test in nitrogen gas.

The specimens subjected to $\Delta P$-constant tests were broken into two parts by additional fatigue loading, and then used for the observation of the fracture surfaces via scanning electron microscope (SEM). On the other hand, $\Delta K$-constant tests were halted before fracture and the specimens were cut along mid-thickness sections, then the lateral surfaces were polished with colloidal $\mathrm{SiO}_{2}$ for the analyses via EBSD and ECCI. EBSD analysis was conducted by using a Schottky type field-emission (FE) SEM operated at $15 \mathrm{kV}$, and the electron beam step size of $0.1 \sim 1.0 \mu \mathrm{m}$ was used for data collection. ECCI was also performed by a FE-SEM with the acceleration voltage of $15 \mathrm{kV}$. Based on crystallographic orientation maps obtained via EBSD, we evaluated the amount of plastic deformation around the crack propagation paths by using two types of strain analyses, i.e. grain reference orientation deviation (GROD) and kernel average misorientation (KAM). Site-specific TEM samples were extracted directly from the fracture surfaces of the $\Delta K$ constant test specimens using a focused ion beam (FIB) machine. The samples were observed using a fieldemission-TEM, operated at $300 \mathrm{kV}$ in dark-field scanning TEM (DF-STEM) mode.

\section{Results and discussion}

\subsection{Macroscopic FCG behavior}

The relationships between FCG rate, $\mathrm{d} a / \mathrm{d} N$ and $\Delta K$ (FCG curve) measured in each testing environment are presented in Fig. 1. The FCG curves in air and nitrogen were linear throughout the tested $\Delta K$ range, and the FCG rate in nitrogen was approximately $70 \%$ slower than in air. On the other hand, clear distinction of Stage I and II crack propagation is visible on the FCG curves in hydrogen gas, demonstrating two important key findings. Firstly, the transitional stress intensities dividing Stage I and II, $\Delta K^{\mathrm{T}}$ shifted to lower values as the pressure of hydrogen gas was increased. Secondly, although the FCG rate at Stage I in $0.7 \mathrm{MPa}$ hydrogen was equivalent with that in air, acceleration or deceleration of FCG was confirmed in Stage I at higher and lower hydrogen gas pressures. Namely, $20 \mathrm{MPa}$ hydrogen resulted in $2 \sim 3$ times accelerated FCG in Stage I, whereas in $0.2 \mathrm{MPa}$ hydrogen, it became slightly slower. In addition to the results of $\Delta P$-constant tests, the FCG rates measured via $\Delta K$-constant tests in air and $0.7 \mathrm{MPa}$ hydrogen were plotted in the same diagram. No detectable difference between the FCG rates in air and 0.7 MPa hydrogen was found in $\Delta K$-constant tests, and the values of FCG rates were almost consistent with those measured in $\Delta P$ constant tests at corresponding $\Delta K$ values. Contrary to Stage I regime where hydrogen had only a mild influence on the macroscopic FCG rates, dramatic acceleration of FCG was confirmed in Stage II with the acceleration rate approximately 30 times compared to the FCG rate in air. Although, the $\Delta K^{\mathrm{T}}$ point had strong dependence on the hydrogen gas pressures, the FCG rates after the transition showed almost the same values in $0.7 \sim 90 \mathrm{MPa}$ hydrogen gas, and were lying on the line corresponding to $\sim 30$ times acceleration.

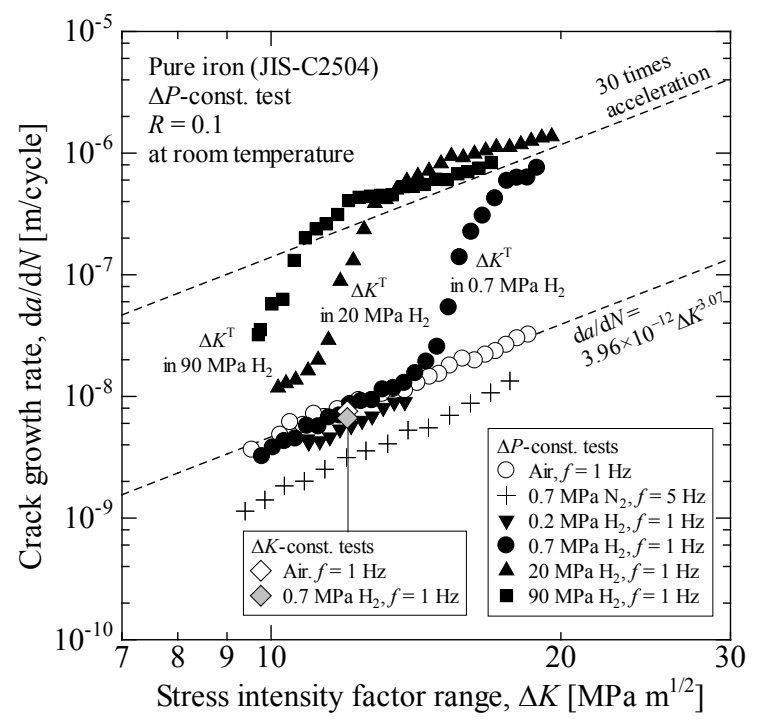

Fig. 1 Relationship between FCG rate, $\mathrm{d} a / \mathrm{d} N$, and stress intensity factor range, $\Delta K$, measured in each environment. 

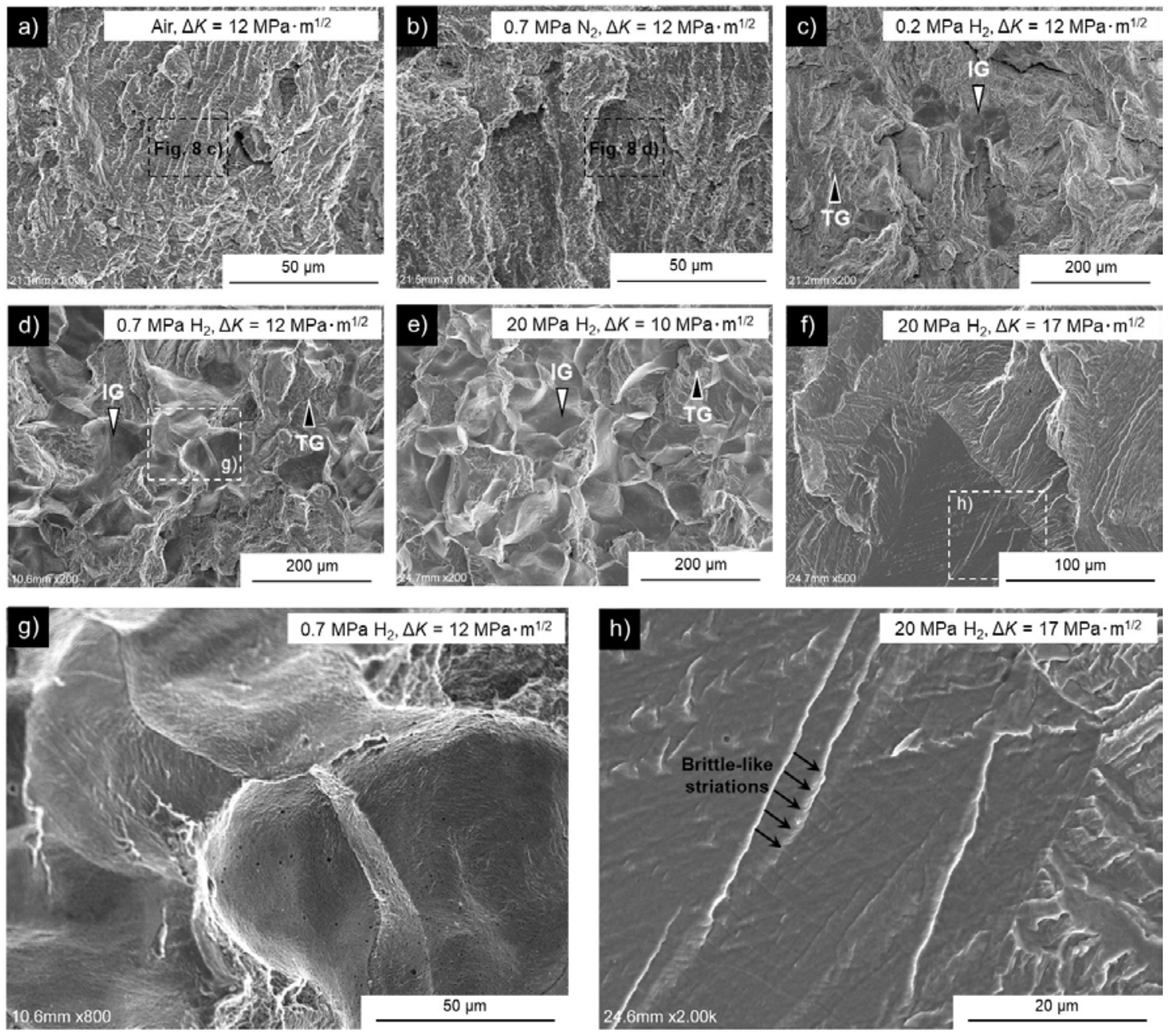

Fig. 2 SEM micrographs of the fracture surfaces formed in each environmental condition with the corresponding $\Delta K$ values of $10 \sim 17 \mathrm{MPa} \cdot \mathrm{m}^{1 / 2} \cdot \mathrm{g}$ ) and $\mathrm{h}$ ) are the magnified views of the regions surrounded by white dotted lines in d) and f) respectively. The crack growth directions are from bottom to top.

\subsection{Fracture surface morphologies}

Figs. 2 (a) (e) present the SEM micrographs of the fracture surfaces corresponding to $\Delta K=10 \sim 12$ $\mathrm{MPa} \cdot \mathrm{m}^{1 / 2}$ in air, nitrogen and $0.2 \sim 20 \mathrm{MPa}$ hydrogen. In the case of air, clear TG striation-like stripe patterns appeared on the whole fracture surface (Fig. 2 (a)), whereas the striations in the case of nitrogen gas became blurred in appearance (Fig. 2 (b)). The spacing of those TG striations was 1 or 2 orders of magnitude larger than the measured FCG rate at the corresponding $\Delta K$ value, which is typical for steels tested under relatively low stress intensity conditions [9,10]. Conversely, the fracture surfaces in hydrogen gas were covered by brittle-like appearance IG facets (Figs. 2 (c) (e)) mixed with the ductile TG fracture features. Moreover, the formation of the IG fracture surface seemed to be more pronounced at higher pressure hydrogen $(20 \mathrm{MPa})$ when we compared Figs. 2 (c) $\sim$ (e) to each other. Figs. 2 (f) and (h) show the fracture surface formed in $20 \mathrm{MPa}$ hydrogen gas at the $\Delta K$ value of $17 \mathrm{MPa} \cdot \mathrm{m}^{1 / 2}$, i.e. Stage II FCG regime. In this case, IG facets which were observed in the lower $\Delta K$ (Fig. 2 (e)) completely disappeared, and the fracture surface was covered by flat QC planes accompanied by brittle-like fatigue striations (Fig. $2(\mathrm{~h})$ ), as observed in the previous papers regarding commercial steels [5-7].

In order to correlate the propensity of IG fracture and the macroscopic FCG rates in Stage I, we examined the area fractions of IG facets on the fracture surfaces and plotted them as the function of $\Delta K$. The results of those examinations are presented in Fig. 3. In this diagram, each plot was constructed according to the three SEM pictures with the magnification of 200 times, which were taken from the positions on the fracture surfaces corresponding to the target $\Delta K$ values. The area fraction of IG reached almost zero at the onset of Stage II crack growth in 0.7 and $20 \mathrm{MPa}$ hydrogen, where the fracture morphology was abruptly replaced by QC type features as shown in Fig. 2 (f). However, the more important finding here is that the fraction of IG fracture surface clearly increased with the increment of hydrogen gas pressure, meaning the macroscopic FCG rate in Stage I was controlled by the propensity of the IG cracking. Specifically, the higher IG fracture ratio resulted in the more accelerated FCG rate. 


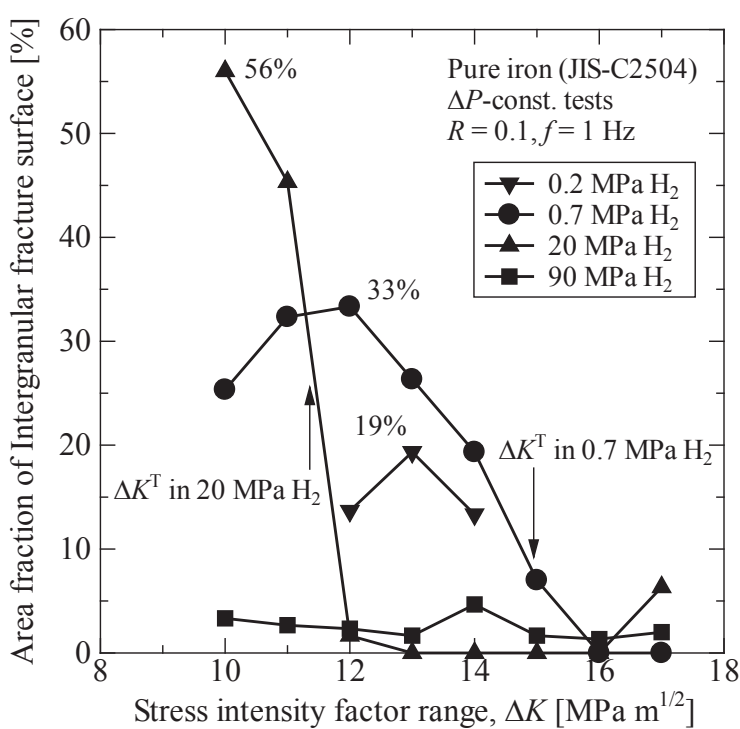

Fig. 3 Area fraction of the IG fracture surface in $0.2,0.7$ and $20 \mathrm{MPa}$ hydrogen gas as the function of stress intensity factor range, $\Delta K$.

\subsection{Dislocation structure underneath the hydrogen-induced IG fracture surface}

Generally, IG fracture surface is one of the representative fractographic features in the hydrogenassisted cracking of high-strength steels [11-13]. In such cases, the crack propagation process is dominated by a relatively brittle manner both in macroscopic and microscopic scale, therefore the FCG rate can be dramatically accelerated even at intermediate testing frequencies such as $f=1 \mathrm{~Hz}$. On the other hand, only a slight acceleration ( $2 \sim 3$ times compared to in air) was detected in case of pure iron (Fig. 1), although similar brittle appearance IG facets were clearly observed on the fracture surfaces (Figs. 2 (c) $\sim($ f)), implying that the microscopic mechanism dominating the IG crack propagation in Stage I FCG in pure iron was not identical with the one in high-strength steels. In Fig. 2 $(\mathrm{g})$, a higher magnification image of the IG fracture surface formed in $0.7 \mathrm{MPa}$ hydrogen is presented. Even though the IG facets seemed brittle in a macroscopic view (Figs. 2 (c) $\sim(\mathrm{e})$ ), numerous trenches and uneven, wavy surfaces can be seen on microscopic scale, indicating the contribution of some plasticity on the fracture process. To elucidate whether this unevenness was the result of dislocation activity or not, a thin-foil sample was directly extracted from the IG fracture surface on the $\Delta K$-constant-tested sample in $0.7 \mathrm{MPa}$ hydrogen, and was then visualized using TEM. The dark-field STEM micrograph of the dislocation structure beneath the IG fracture surface in $0.7 \mathrm{MPa}$ hydrogen at $\Delta K=12 \mathrm{MPa} \cdot \mathrm{m}^{1 / 2}$ is indicated in Fig. 4 (b) together with a SEM picture of the location for the TEM sample extraction (Fig. 4 (a)). The dislocations were organized into cellular structures with clear and thin cell walls, indicating that the final separation of the grain boundary was mediated by a high level of plastic strain.
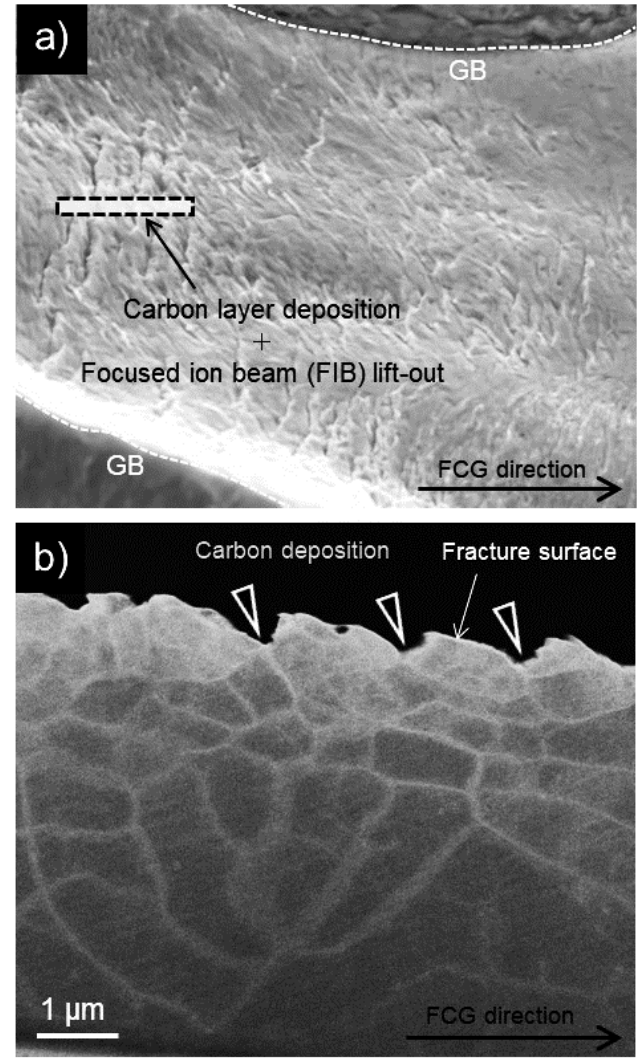

Fig. 4 (a) SEM micrograph of the IG fracture surface formed in $0.7 \mathrm{MPa}$ hydrogen at $\Delta K=12 \mathrm{MPa} \cdot \mathrm{m}^{1 / 2}$ from which the thinfoil sample was extracted. (b) Dark field STEM image of the dislocation structure immediately beneath the IG fracture surface shown in (a).

\subsection{Deformation structure analyses around the crack propagation paths via EBSD and ECCI}

For the quantitative evaluation of the amount of plastic deformation around the crack propagation paths in air and hydrogen in Stage I FCG, the specimens subjected to $\Delta K$-constant tests in air and $0.7 \mathrm{MPa}$ hydrogen gas were cut along mid-thickness sections and the surfaces were then observed via EBSD. Figs. 5 (a) and (b) present the crystallographic orientation maps along the midthickness fracture paths in air and $0.7 \mathrm{MPa}$ hydrogen at $\Delta K=12 \mathrm{MPa} \cdot \mathrm{m}^{1 / 2}$. In these EBSD analyses, the beam step size of $1.0 \mu \mathrm{m}$ was used. The crack propagation in air was almost fully $\mathrm{TG}$, on the other hand, in $0.7 \mathrm{MPa}$ hydrogen, some parts of the fracture path were IG (indicated by arrowheads), as was confirmed in the fracture surface examination (Fig. 2 (d)). In Figs. 5 (c) (f), the GROD and KAM maps which were constructed from Figs. 5 (a) and (b) are presented. Significant extent of plastic deformation can be seen around the fracture path in the case of air (Figs. 5 (c) and (e)). Likewise, severe plasticity was also detected in hydrogen both around the TG and IG fractured regions, which was in accordance with the observation result using TEM (Fig. 4 (b)). Fig. 6 shows the histograms of the GROD and KAM values constructed from the raw EBSD-scanned data indicated in Figs. 5 (c) $\sim$ (f). According to this diagram, both of the GROD and KAM values in $0.7 \mathrm{MPa}$ hydrogen gas surprisingly showed slightly higher 

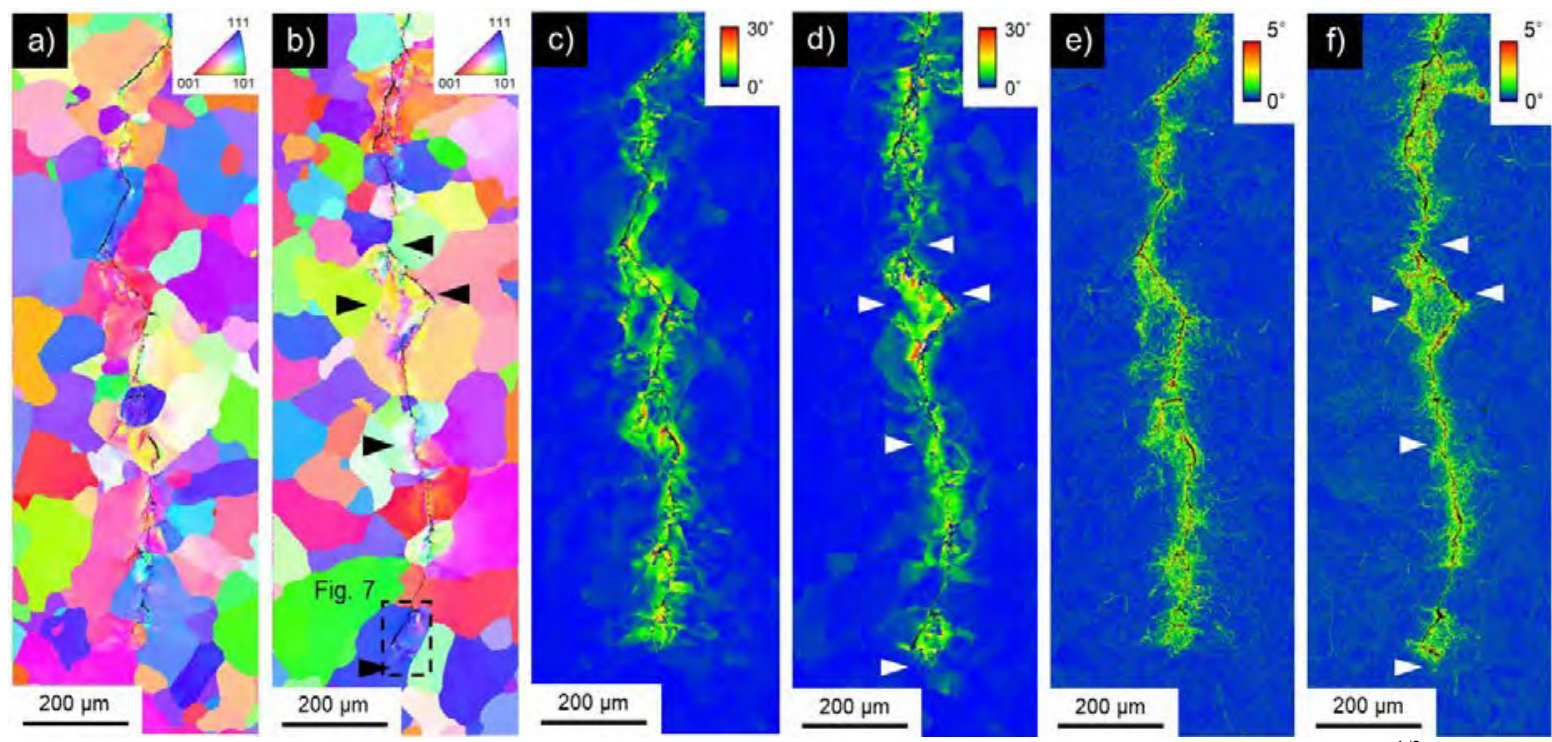

Fig. 5 Mid-thickness fracture paths in (a)(c)(e) air and in (b)(d)(f) $0.7 \mathrm{MPa}$ hydrogen gas at $\Delta K=12 \mathrm{MPa} \cdot \mathrm{m}^{1 / 2}$ analyzed via EBSD. (a)(b), (c)(d) and (d)(e) are the crystallographic orientation maps, GROD maps and KAM maps respectively. The IG fracture paths observed in hydrogen gas are marked with black and white arrowheads in (b), (d) and (f). The crack growth directions are from top to bottom.

misorientation angles than in air, meaning the crack propagation process in hydrogen was accompanied by more intense plastic deformation. In the second part (Part II) of this series of papers, it will be showed that hydrogen has the role to suppress the plasticity expansion around the crack tip region and enhances the fracture along $\{100\}$ type cleavage plane in Stage II FCG regime, resulting in the formation of QC type features (c.f. Fig. 3 (f)) [3]. However, it is worth noting that the above-noted results imply the completely opposite role of hydrogen in Stage I FCG regime. Specifically, when the stress intensity level is below the critical stress intensity to trigger cleavage-like fracture along $\{100\}$ plane $\left(\Delta K<\Delta K^{\mathrm{T}}\right)$, hydrogen accelerates the crack-tip plasticity rather than suppressing it.

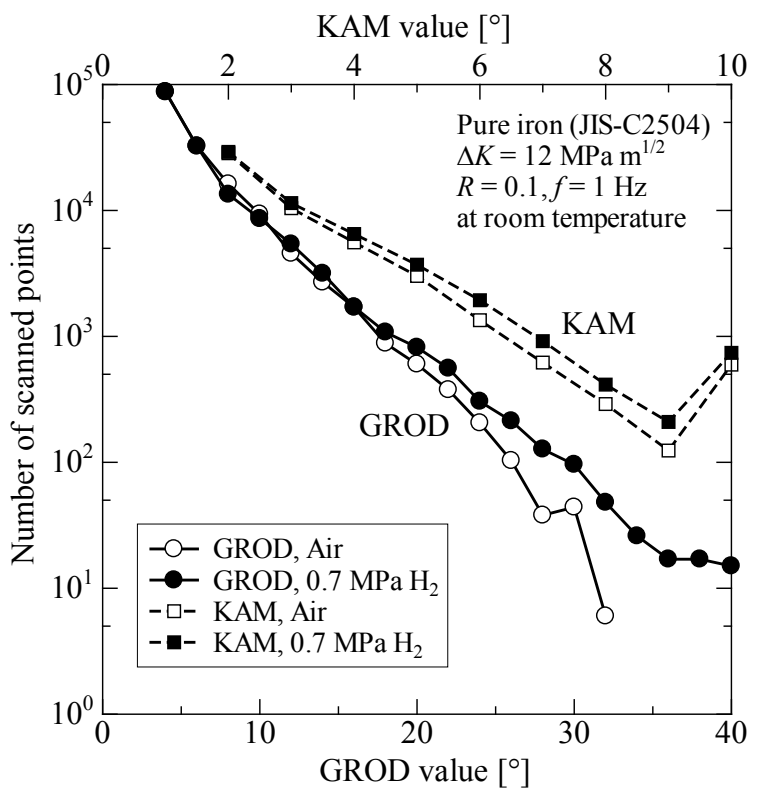

Fig. 6 Histograms of the GROD and KAM values measured in the EBSD analyses shown in Fig. 5.
Our present result regarding Stage I, i.e. the enhancement of plasticity under the presence of hydrogen, is consistent with the results reported by Wang et al. [14], who found that the size of dislocation cells in hydrogen-charged pure nickel subjected to highpressure torsion (HPT) became smaller compared to non-charged material. More recently, they also examined the dislocation structures immediately beneath the IG fracture surface formed in the FCG test of a structural carbon steel tested in $40 \mathrm{MPa}$ hydrogen gas, and reported that the dislocations showed smaller cell diameters compared to the cells formed beneath ductile striations in air [15]. Throughout the series of publications, they explained such alteration of dislocation behavior based on the framework of hydrogen-enhanced localized plasticity (HELP) hypothesis [16-18]. According to HELP assertion, hydrogen distributed around the dislocation stress field reduces the elastic interaction of dislocations with other obstacles, thereby enhancing their mobility and velocity $[17,18]$, as well as hydrogen in normal interstitial lattice sites also reduces the nucleation energy of dislocations $[19,20]$. Wang et al. assumed that this role of hydrogen, i.e. easing dislocation generation and mobility, can result in the finer and more evolved dislocation structures under the presence of hydrogen [14,15], which seems to be applicable also in the case of severely strained region ahead of the fatigue crack tip in pure iron.

Fig. 7 shows the high-mag. images around the crack tip region in $0.7 \mathrm{MPa}$ hydrogen obtained via EBSD (beam step size of $0.1 \mu \mathrm{m}$ ) and ECCI. In this region, the crack propagated originally through TG path (indicated by upward arrows in Figs. 7 (a) (c)) and then changed its path along the GB (indicated by downward arrows in Figs. 7 (a) (c)). As shown in the low-mag. observation (Fig. 5), the plastic strain visualization via EBSD (Figs. 7 (b) and (c)) demonstrated that severe plastic deformation was operated in the adjacent grains 

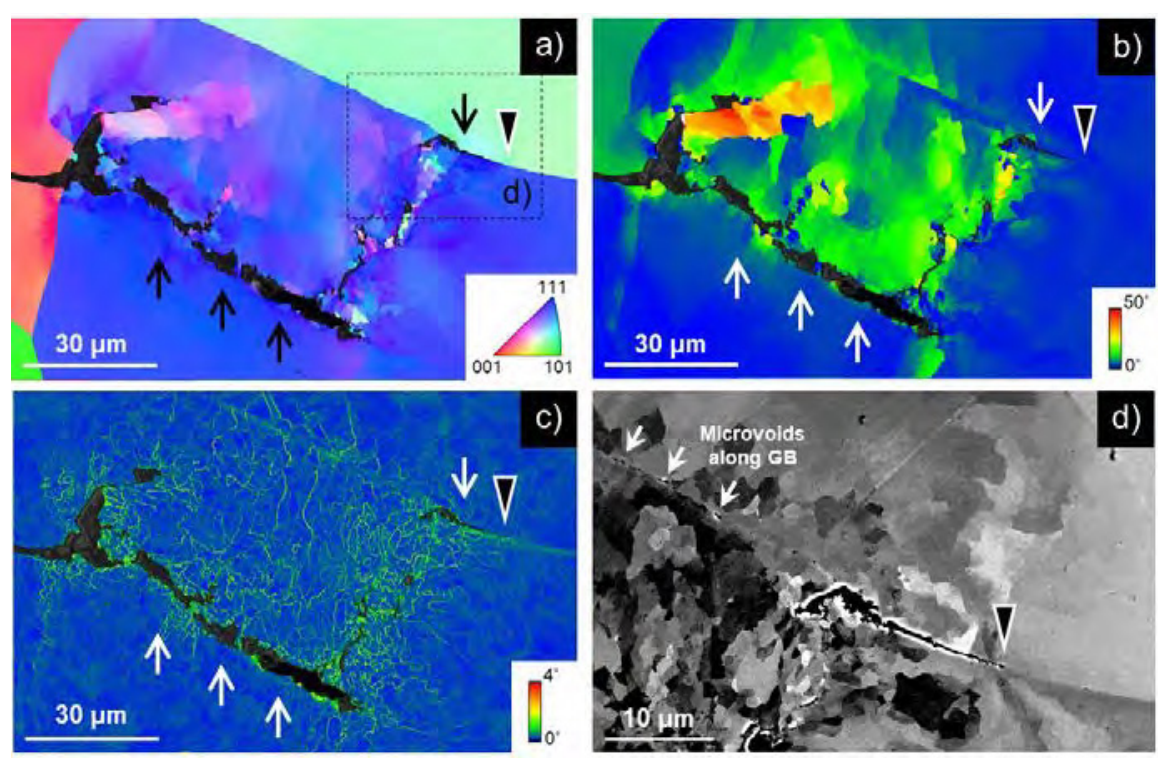

Fig. 7 High-mag. observation around the tip of fatigue crack propagated in $0.7 \mathrm{MPa}$ hydrogen gas at $\Delta K=12 \mathrm{MPa} \cdot \mathrm{m}^{1 / 2}$. (a), (b) and (c) are the crystal orientation, GROD and KAM maps respectively analyzed via EBSD. (d) is the ECC image of the region surrounded by black dotted line in (a). The crack growth directions are from left to right.

surrounding the IG crack propagation path, also demonstrating the clear contrast changes in the corresponding ECC image (Fig. 7 (d)). However, it is worth noting that some microvoids were generated along the GB, where the evolved dislocation structure bumped into it as shown in Fig. 7 (d) with white arrows. Combining all the above, we can assume the following sequences for IG cracking during Stage I HAFCG:

1. Firstly, numerous dislocations are emitted from the tip of transgranularly propagating fatigue crack, and severe plastic strain ahead of the crack tip organizes them into cellular structure. As the cell structure evolution proceeds, more dislocations are absorbed into the cell walls and annihilate each other, which makes the cell walls thinner and results in the formation of subgrain boundaries. Moreover, both of the dislocation emission [19-21] and evolution [14,15,22] processes are accelerated by hydrogen. These modifications of dislocation behavior raise higher misorientation angles in GROD and KAM analyses of the sample tested in hydrogen gas (Fig. 6).

2. Secondly, if some GBs are located near the propagating crack tip region, dislocations pile-up onto the GBs, leading to the local stress concentration and formation of some lattice defects such as vacancies due to the dislocations-GB interactions, i.e. disordering of the GB structure. It is commonly accepted that hydrogen in metals potentially stabilizes the point defects and enhances the generation of non-thermal equilibrium superabundant vacancies as reported by Nagumo et al. $[23,24]$, demonstrating a possibility that the agglomeration of those stabilized vacancies can result in the nucleation of microvoids along the GBs. Recently, Lawrence et al. investigated the density of vacancies and vacancy clusters in hydrogen pre-charged single crystalline and polycrystalline pure nickel after tensile deformation by means of thermal desorption spectroscopy (TDA) and positron annihilation spectroscopy (PAS), and reported that polycrystalline material showed higher peak temperature in TDS and longer positron lifetime in PAS [25]. Their results strongly suggest that the GB itself - or the volumes adjacent to them - are the preferential sites for hydrogen-induced vacancies or vacancy cluster formation, rather than grain interior, which is in accordance with our present observation results (Fig. 7).

3. Finally, when sufficient numbers of GB microvoids are formed, their coalescence trigger the final separation of the GBs, and propel the crack tip ahead.

Referring to the TEM micrograph shown in Fig. 4 (b), we can observe the asperity formed on the IG fracture surface, which may be caused by the microvoids formation and coalescence processes. Furthermore, each pit of the troughs accurately corresponds to the positions at which the dislocation cell walls intersect with the fracture surface (indicated by black arrowheads), further supporting our assumption that dislocations-GB interaction is the main reason in triggering the microvoiding near GB regions. On the other hand, a slightly different mechanism has been proposed by Wang et al. [26] and others [16,22] to explain the IG fracture of hydrogen-charged pure nickel and pure iron subjected to monotonic tensile tests. In their assumption, the combined effect of HELP and the hydrogenenhanced decohesion (HEDE) model [27] play a crucial role in causing the fracture along GBs. According to the HELP framework, the hydrogen atoms - which distribute inside the grains - are transported by moving dislocations [28,29] and then deposited into GBs in conjunction with the dislocations piling-up onto GBs. This process achieves the local stress concentration and hydrogen agglomeration around GBs, which, theoretically, is enough to satisfy the critical condition to operate atomistic decohesion $[16,22,26]$. Additionally, they also found that the development level of the dislocation structure underneath the IG fracture surface 
far exceeds the one under the absence of hydrogen at an equivalent strain level [22,26], as per the recent investigation conducted by Wang et al. $[14,15]$. Martin et al. suggested that the work hardening associated with such hydrogen-enhanced plasticity evolution has a role in assisting GB separation by rising the local stress level to exceed the ideal GB cohesive force [22]. Likewise, in our study, the higher GROD and KAM values in hydrogen demonstrated similar enhancement of plasticity, possibly resulting in the further hardening of the matrix near the crack tip region. Specifically, both the microvoid coalescence model and HELP-mediated HEDE model proposed by Martin et al. [22] may have contributed to the GB separation in Stage I, although the latter was not directly confirmed in the present study.

\subsection{Comparison of the TG fracture morphology formed in air, nitrogen and hydrogen gas}

If the IG fracture in HAFCG of pure iron is dominated by the aforementioned two mechanisms, i.e. GB microvoiding model and the model by Martin et al. [22], the propensity of IG cracking should show the dependency on the hydrogen concentration inside the material. This hypothesis can reasonably explain the dependency of IG fracture ratio and corresponding FCG rate on the testing hydrogen gas pressure as indicated in Fig. 3. Namely, higher hydrogen gas pressure, i.e. higher hydrogen concentration in the material, increased the propensity for IG fracture which resulted in an accelerated FCG rate in $20 \mathrm{MPa}$ hydrogen gas. However, one query for such an assumption is the slightly slower FCG rate measured in $0.2 \mathrm{MPa}$ hydrogen gas compared to that in air (Fig. 1). When attempting to explain this mysterious phenomenon, we have to consider both positive and negative effects of gaseous hydrogen environment for the FCG processes. Here, the positive effect represents the extremely low content of impurities, such as oxygen or water vapor in the hydrogen gas used for the experiments. It is understood that these components have a strong influence on the FCG rates of steels or aluminum alloys, and there already exist numerous studies which mention their deleterious aspect on the FCG behavior [30,31]. Additionally, it was also reported that the presence or absence of oxygen and water vapor cause a significant change of fatigue striation morphology on the fracture surface [30]. In general, the fatigue striations in non-aggressive environments are formed through the repetition of normal slip-off in loading processes and reverse-slip during unloading processes [32]. McEvily et al. found that the striations of Type304 stainless steel in a vacuum environment become more blurred in appearance compared to those formed in air, and discussed these differences with reference to the crack blunting process [30]. Specifically, the absence of adsorption of oxygen or water vapor on the bare surface in crack tip facilitates larger crack blunting and vanishes the border of striations formed in prior loading cycles. Likewise, in our study, these two aspects, i.e. crack deceleration (Fig. 1) and vanishing of clear striations (Fig. 2 (b)) were observed in the case of crack propagation in $0.7 \mathrm{MPa}$ nitrogen gas. For comparison, in Fig. 8, the detailed appearance of the fracture surfaces formed in air, 0.7 $\mathrm{MPa}$ nitrogen and $0.7 \mathrm{MPa}$ hydrogen gas at $\Delta K=12$ $\mathrm{MPa} \cdot \mathrm{m}^{1 / 2}$, are presented. The high magnification image in Fig. 8 (b) indicates the region surrounded by white dotted lines in the overview image in Fig. 8 (a), and Figs. 8 (c) and (d) are the details of the areas surrounded by black dotted lines in Figs. 2 (a) and (b), respectively. When comparing Figs. 8 (b) and (d), the TG part of the fracture surface in $0.7 \mathrm{MPa}$ hydrogen (Fig. 8 (b)) shows an obvious similarity to the fracture surface formed in $0.7 \mathrm{MPa}$ nitrogen gas (Fig. $8(\mathrm{~d})$ ); however, they prove to be different from the TG striations formed in air (Fig. 8 (c)). This similarity of fracture surface appearance strongly suggests that the TG crack path in hydrogen gas at Stage I has a role in decelerating the FCG rate compared to in air, whereas IG fracture surface enhances the faster crack propagation. In summary, at lower pressure $(0.2 \mathrm{MPa})$, the positive effect exceeded the negative effect, while such effects balanced out in the case of $0.7 \mathrm{MPa}$ hydrogen.

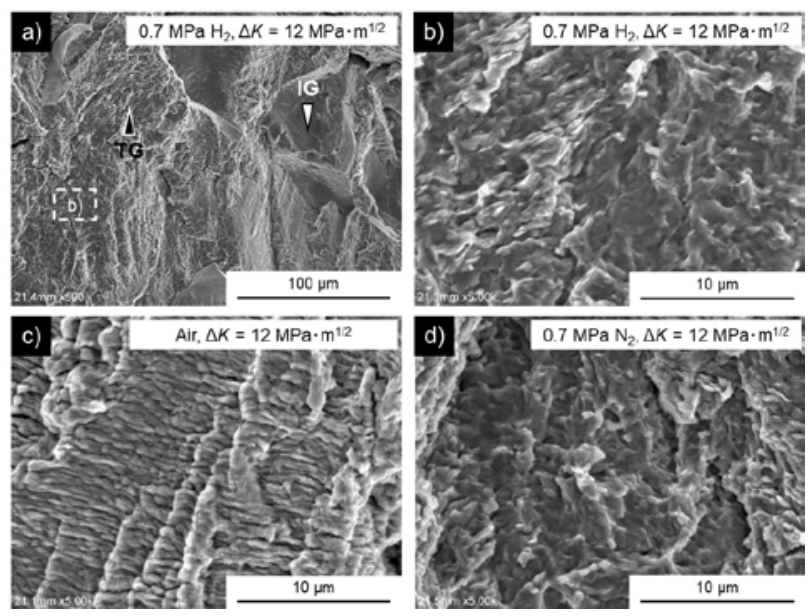

Fig. 8 SEM micrographs of the fracture surfaces formed in (a)(b) 0.7 MPa hydrogen gas, (c) air and (d) $0.2 \mathrm{MPa}$ nitrogen gas at the corresponding $\Delta K$ value of $12 \mathrm{MPa} \cdot \mathrm{m}^{1 / 2}$. (b) shows the magnified view of the area surrounded by a white dotted line in (a). (c) and (d) correspond to the regions surrounded by black dotted lines in Fig. 2 (a) and (b), respectively. The crack growth directions are from bottom to top.

\section{Conclusions}

1. FCG behavior in hydrogen gas was clearly divided into two distinctive regimes, i.e. relatively low stress intensity regime with no or slight acceleration of FCG rate compared to in air (Stage I), and higher stress intensity regime with dramatic acceleration of FCG (Stage II). Fracture surface observation revealed that hydrogen triggers the fracture along grain boundaries (GB) in Stage I FCG, whereas in Stage II, the fracture was characterized by a quasi-cleavage (QC) type feature.

2. The FCG rates measured in Stage I showed considerable dependency on the testing hydrogen gas pressures. Namely, high pressure, e.g. $20 \mathrm{MPa}$ resulted in $2 \sim 3$ times acceleration and low pressure, e.g. 0.2 $\mathrm{MPa}$ resulted in a slightly slower FCG compared to that 
in air. While at intermediate pressure, e.g. $0.7 \mathrm{MPa}$, the FCG rate was almost equivalent to that measured in air.

3. The area fraction of IG fracture surface in Stage I is also dependent on the testing hydrogen gas pressure as is the case with the measured FCG rate. This result indicated that increased hydrogen gas pressure, i.e. hydrogen content inside the material, enhances the propensity for IG cracking and subsequent FCG acceleration in Stage I.

4. TEM and EBSD analyses demonstrated significant plasticity around the IG fracture paths in hydrogen gas, meaning that the IG fracture in Stage I FCG is not just a brittle type fracture. Additionally, ECCI observation revealed the microvoiding along GBs near the propagating crack tip, indicating that hydrogen-modified dislocation behavior, and its interaction with GBs, plays a crucial role in triggering the IG cracking.

5. In low pressure hydrogen, e.g. $0.2 \mathrm{MPa}$, and 0.7 $\mathrm{MPa}$ nitrogen gas, the measured FCG rates showed considerably slower values compared to those in air. Even though hydrogen facilitates GB cracking on some parts on the fracture surface, other parts of the fracture surface in hydrogen, i.e. TG region, exhibited a definite similarity with the fracture surface formed in nitrogen gas. This result implies that TG crack paths in hydrogen have a role in decelerating FCG rates due to the absence of oxygen and water vapor as is the case in nitrogen gas. The macroscopic FCG rate in hydrogen gas is determined by the competition process of this positive aspect (TG cracking) and negative aspect (IG cracking).

This work was supported by JSPS KAKENHI Grant Number JP16H04238 and JP16J02960. The Research Council of Norway is acknowledged for its support through the Norwegian Center for Transmission Electron Microscopy, NORTEM (197405/F50). This study also forms part of the "HIPP" project from the PETROMAKS2 program, funded by the Research Council of Norway [Grant Number: 102006899].

\section{References}

[1] H.J. Cialone, J.H. Holbrook, Metall. Trans. A 16 (1985) 115-122.

[2] B.P. Somerday, P. Sofronis, K.A. Nibur, C. San Marchi, R. Kirchheim, Acta Mater. 61 (2013) 6153-6170.

[3] D. Birenis, Y. Ogawa, H. Matsunaga, O. TakakuwaJ. Yamabe, Ø. Prytz, A. Thøgersen, to be presented in 12th International Fatigue Congress (Fatigue 2018).

[4] A.J. Slifka, E.S. Drexler, N.E. Nanninga, Y.S. Levy, J.D. McColskey, R.L. Amaro, A.E. Stevenson, Corros. Sci. 78 (2014) 313-321.

[5] Y. Ogawa, H. Matsunaga, J. Yamabe, M. Yoshikawa, S. Matsuoka, Int. J. Fatigue 103 (2017) 223-233.

[6] Y. Takahashi, H. Nishikawa, Y. Oda, H. Noguchi, Mater. Lett. 64 (2010) 2416-2419.

[7] T.J. Marrow, P.J. Cotterill, J.E. King, Acta Metall. Mater. 40 (1992) 2059-2068.
[8] ASTM, 2010, E647-08-1. West Conshohocken: ASTM International.

[9] H. Cai, A.J. McEvily, Mater. Sci. Eng. A 314 (2001).

[10] A.J. McEvily, H. Matsunaga, Sci. Iran. B Mech. Eng. 17 (2010) 75-82.

[11] Z. Sun, G. Benoit, C. Moriconi, F. Hamon, D. Halm, F. Hamon, G. Hénaff, Int. J. Hydrogen Energy 36 (2011) 8641-8644.

[12] J. Yamabe, T. Matsumoto, S. Matsuoka, Y. Murakami, Int. J. Fract. 177 (2012) 141-162.

[13] C.J. McMahon, Eng. Fract. Mech. 68 (2001) 773-788.

[14] S. Wang, A. Nagao, K. Edalati, Z. Horita, I.M. Robertson, Acta Mater. 135 (2017) 96-102.

[15] S. Wang, A. Nagao, P. Sofronis, I.M. Robertson, Acta Mater. 144 (2018) 164-176.

[16] I.M. Robertson, P. Sofronis, A. Nagao, M.L. Martin, S. Wang, D.W. Gross, K.E. Nygren, Metall. Mater. Trans. B 46 (2015).

[17] I.M. Robertson, Eng. Fract. Mech. 68 (2001) 671-692.

[18] H.K. Birnbaum, P. Sofronis, Mater. Sci. Eng. A 176 (1994) 191-202.

[19] A. Barnoush, H. Vehoff, Acta Mater. 58 (2010) 5274-5285.

[20] A. Barnoush, H. Vehoff, Scr. Mater. 55 (2006) 195-198.

[21] S. Lynch, Corros. Rev. 30 (2012) 105-123.

[22] M.L. Martin, B.P. Somerday, R.O. Ritchie, P. Sofronis, I.M. Robertson, Acta Mater. 60 (2012).

[23] M. Nagumo, Mater. Sci. Technol. 20 (2004) 940-950.

[24] K. Takai, H. Shoda, H. Suzuki, M. Nagumo, Acta Mater. 56 (2008) 5158-5167.

[25] S.K. Lawrence, Y. Yagodzinskyy, H. Hänninen, E. Korhonen, F. Tuomisto, Z.D. Harris, B.P. Somerday, Acta Mater. 128 (2017) 218-226.

[26] S. Wang, M.L. Martin, P. Sofronis, S. Ohnuki, N. Hashimoto, I.M. Robertson, Acta Mater. 69 (2014) 275-282.

[27] R.A. Oriani, P.H. Josephic, Acta Metall. 22 (1974) 1065-1074.

[28] J. Tien, A.W. Thompson, I.M. Bernstein, R.J. Richards, Metall. Trans. A 7 (1976) 821-829.

[29] M. Dadfarnia, M.L. Martin, A. Nagao, P. Sofronis, I.M. Robertson, J. Mech. Phys. Solids 78 (2014) 511-525.

[30] A.J. McEvily, J.L. Gonzalez Velazquez, Metall. Trans. A 23 (1992) 2211-2221.

[31] N.M. Grinberg, Int. J. Fatigue 4 (1982) 83-95.

[32] R. Pippan, C. Zelger, E. Gach, C. Bichler, H. Weinhandl, Fatigue Fract. Eng. Mater. Struct. 34 (2011). 